\title{
Chemical evolution models for NGC 6822 using planetary nebulae abundances
}

\author{
Liliana Hernández-Martínez ${ }^{1,2}$, Leticia Carigi ${ }^{1}$, Miriam Peña $^{1}$ \\ and Manuel Peimbert ${ }^{1}$ \\ ${ }^{1}$ Instituto de Astronomía, Universidad Nacional Autónoma de México, Apdo. Postal 70264, \\ Méx. D. F., 04510 México. (carigi, miriam, peimbert@astro.unam.mx). \\ ${ }^{2}$ Instituto Nacional de Astrofísica, Optica y Electrónica, Luis Enrique Erro No. 1, Puebla, \\ México. (lilihe@inaoep.mx).
}

\begin{abstract}
We present chemical evolution models for the dwarf irregular NGC 6822, using chemical abundances of Planetary Nebulae (PNe) and HII regions and also the mass of gas $\left(\mathrm{M}_{\text {gas }}\right)$ as observational constraints. Chemical evolution models have been calculated to reproduce the abundances as derived from both, collisionally excited lines (CELs) and recombination lines (RLs). In our models, the chemical contribution of low and intermediate mass stars (LIMS) is time delayed, while for the massive stars the chemical contribution is instantaneous, as in Franco \& Carigi (2008). The chemical contribution of SNIa is included in our model, thus we are also able to reproduce the observational $\mathrm{Fe} / \mathrm{H}$ abundance obtained from A stars.
\end{abstract}

Keywords. Galaxies: abundances, galaxies: evolution, galaxies: individual (NGC 6822), HII regions, planetary nebulae: general

\section{Introduction}

Planetary Nebulae (PNe) constitute one of the most valuable chemical tracers of the past abundances in the interstellar medium (ISM). Then PN characteristics are important as observational constraints in chemical evolution models, allowing us to improve the inferred chemical history (Hernández-Martínez et al. 2009). We constructed a chemical evolution model similar to that proposed by Franco \& Carigi (2008) with a time-delay prescription for the chemical enrichment produced by LIMS. This time-delay term makes these stars bring out the nuclear processed material to the ISM at a single time after the star formation, while the contribution of massive stars (MS) is instantaneous, like in the instantaneous recycling approximation (IRA) models (Talbot \& Arnett 1971). In Hernández-Martínez et al. (2011) we present several models computed to reproduce the abundances obtained from CELs and from RLs, trying to discriminate between both methods.

\section{Models}

We computed chemical evolution models in which the lower mass limit of the IMF was fixed at $0.1 \mathrm{M}_{\odot}$ and the upper mass limit, $M_{\text {up }}\left(=40 \rightarrow 80 \mathrm{M}_{\odot}\right)$, is a free parameter adjusted to reproduce the observed $\mathrm{O} / \mathrm{H}$ abundance ratios in $\mathrm{H}$ II regions (from CELs or RLs, both corrected by dust depletion). We also include a well mixed outflow, where we assume that the ejecta of MS, LIMS, and SNIa are well mixed with the ISM of the galaxy before it is expelled to the intergalactic medium. Our models require a well mixed outflow, starting at $t=1.2$ Gyr (the time when the SFR starts) throughout 5.1 Gyr to reproduce the actual $M_{\text {gas }}$ in the galaxy. 
a)

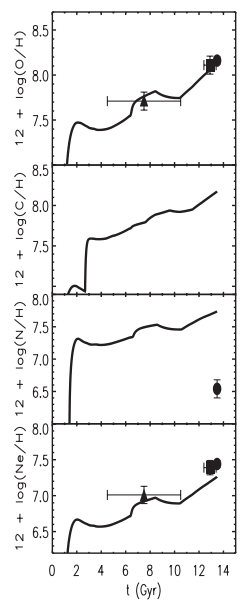

b)

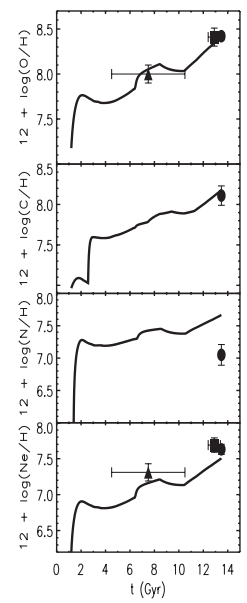

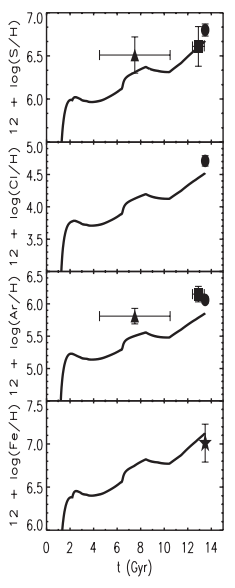

Figure 1. Chemical evolution of NGC 6822 as predicted by a) Model M4C (for CEL abundances) and b) Model M1R (for RL abundances). The circles, squares and triangles are the observational values for H II regions, young and old PN populations, respectively. The star in the $\mathrm{Fe} / \mathrm{H}$ panel is the value of iron $(\mathrm{Fe} / \mathrm{H})$ from A-type stars as given by Venn et al. (2001).

Figure 1 shows two chemical evolution models for NGC 6822. M4C reproduces abundances obtained from CELs and M1R reproduces abundances obtained from RLs. These models reproduce a similar number of observational constraints, in particular both reproduce the evolution history of the $\mathrm{O} / \mathrm{H}$ abundance (as given by $\mathrm{HII}$ regions and young and old $\mathrm{PNe}$ ) and also some abundance ratios such as $\log (\mathrm{C} / \mathrm{H}), \log (\mathrm{Ne} / \mathrm{H}), \log (\mathrm{S} / \mathrm{H})$, $\log (\mathrm{Cl} / \mathrm{H}), \log (\mathrm{Ar} / \mathrm{H})$ and $\log (\mathrm{Fe} / \mathrm{O})$. They both fail in reproducing $\mathrm{N} / \mathrm{O}$ ratio, predicting $\log \mathrm{N} / \mathrm{O} \sim 0.6$ higher than the observed values. In all the models we assumed that $1 \%$ of the stars with $\mathrm{M}=3$ and $15 \mathrm{M}_{\odot}$ give up binary systems and every one of these becomes a SNIa. Using this fraction, we can reproduced the abundance of $[\mathrm{Fe} / \mathrm{H}]=-5.04$ dex from A-type stars by Venn et al. (2001). The model M1R is more conservative in the mass of the most massive star formed in NGC $6822\left(M_{\text {up }}=80 \mathrm{M}_{\odot}\right.$, similar to the value derived for the solar vicinity) while $\mathrm{M} 4 \mathrm{C}$ required a much lower $M_{\mathrm{up}}=40 \mathrm{M}_{\odot}$.

\section{Conclusions}

We have constructed a chemical evolution model with a time delayed contribution for LIMS. Our model also include the SNIa contribution to the chemical enrichment in the galaxy. We calculate the chemical evolution of NGC 6822 and at this point we are not able to distinguish, through chemical evolution models, which method for determining abundances (CELs or RLs) is more confident. The main characteristics of the model for NGC 6822 and other results are presented in Hernández-Martínez et al. (2011).

\section{References}

Franco, I. \& Carigi, L. 2008, RMAA, 44, 311.

Hernández-Martínez, L., Peña, M., Carigi, L., \& García-Rojas, J., 2009, A\&A, 505, 1027

Hernández-Martínez, L., Carigi, L., Peña, M., \& Peimbert, M., 2011, A\&\&A, (in press)

Talbot, R. J. Jr. \& Arnett, W. D., 1971, ApJ, 170, 409.

Venn, K. A., Lennon, D. J., \& Kaufer, A., 2001, ApJ, 625, 754. 